\title{
Sorption of Anthraquinone Disperse Dyes by Nylon 6
}

\author{
Takao Shibusawa ${ }^{* 1}$ and Takashi Nakamura*2 \\ *1 Faculty of Engineering, Gunma University, 1-5-1, Tenjin-cho, Kiryu-shi, Gunma, 376 Japan \\ *2 Textile Research Institute of Gunma Prefecture, 5-46-1, Aioi-cho, Kiryu-shi, Gunma, 376 \\ Japan
}

\begin{abstract}
Sorption isotherms of eleven purified anthraquinone disperse dyes on Nylon 6 film from water were measured at 60 and $80^{\circ} \mathrm{C}$. Analysis of the isotherms indicates that two concurrent modes of sorption are operative in the dye sorption; Nernst type partitioning and a Langmuir sorption. Normally, the amount of dye sorbed by the Langmuir sorption $\left(C_{1}\right)$ was much smaller than that $\left(C_{p}\right)$ by the partitioning. However, the ratio $C_{L} /\left(C_{P}+C_{L}\right)$ at saturated dye concentration in water, which is a measure to indicate the contribu. tion of Langmuir sorption to the total dye sorption, increased with an increase in the number of functional group capable of forming intermolecular hydrogen bond in dye. These findings suggest that the hydrogen bonding between the dyes and Nylon 6 plays an important role in the Langmuir sorption.
\end{abstract}

\section{Introduction}

The sorption of some nonionic dyes by polymers from water is satisfactorily described in terms of a dual-mode sorption model expressed by Eq. $1[1,2]$. In this model. two concurrent modes of dye sorption i. e., Nernst type partitioning and a Langmuir sorption are operative.

$$
C_{f}=C_{P}+C_{L}=K_{P} C_{S}+\frac{K_{L} S C_{S}}{1+K_{L} C_{S}}
$$

where $C_{f}$ and $C_{S}$ are the equilibrium dye concentra. tion on polymer and in solution. $C_{p}$ and $C_{l}$. are the equilibrium dye comcentration on polymer sorbed by Nernst type partitioning and Langmuir sorption, respectively. $S$ is the saturation value for Langmuir sorption. $K_{\mathrm{P}}$ and $\mathrm{K}_{\mathrm{L}}$ are the partition coefficient and the Langmuir constant, respectively.

In the sorption of nonionic azo dyes by Nylon 6 from water, partitioning is normally predominant, i. e., $\mathrm{C}_{\mathrm{l}}$ is much smaller than $C_{P}$ except in the range of very low $C_{S}$ [1.2]. The ratio $C_{L} / C_{f}$ at saturated dye concentration in water may be used as a measure to represent the con. tribution of the Langmuir sorption to the total dye sorption (2). In the sorption of azo disperse dyes by some polymers, $C_{L} / C_{f}$ increases with increasing number of functional group capable of intermolecular hydrogen bonding (H-bonding) in dye (2]. This finding suggests that the $\mathrm{H}$-bonding between the azo dyes and polymer substrates plays an important role in the Langmuir sorption.

In the present paper, the experimental sorption isotherms of anthraquinone disperse dyes on nylon 6 from water are analyzed with respect to the capability of substituents in the dyes to form inter-molecular $\mathrm{H}$. bonding.

\section{Experimental}

\subsection{Materials}

The structure of dyes used are shown in Table 1 . Dyes 1-4,6 and 7 were purchased from Tokyo Chemical Industry Co. Ltd. as reagent grade. Dyes 5,8 and 9 were purchased as commercial dye. Dyes 10 and 11 were synthesized from 1-aminoanthraquinone and ethyleneoxide, and leuco-qunizarine and ethanol amine, respectively. They were purified as described previously [2].

Nylon $6 \mathrm{film}$ : the film used was identical as used in previous study [2].

\subsection{Measurements}

Sorption isotherms were measured as described previously [2] except for Dye 6. Since Dye 6 tends to sublimate from aqueous solution and then it is sorbed by silicone rubber plug, nylon 6 film was dyed with Dye 6 in a test tube equipped with ground glass stopper. 
Table 1 Structure of Dyes Used

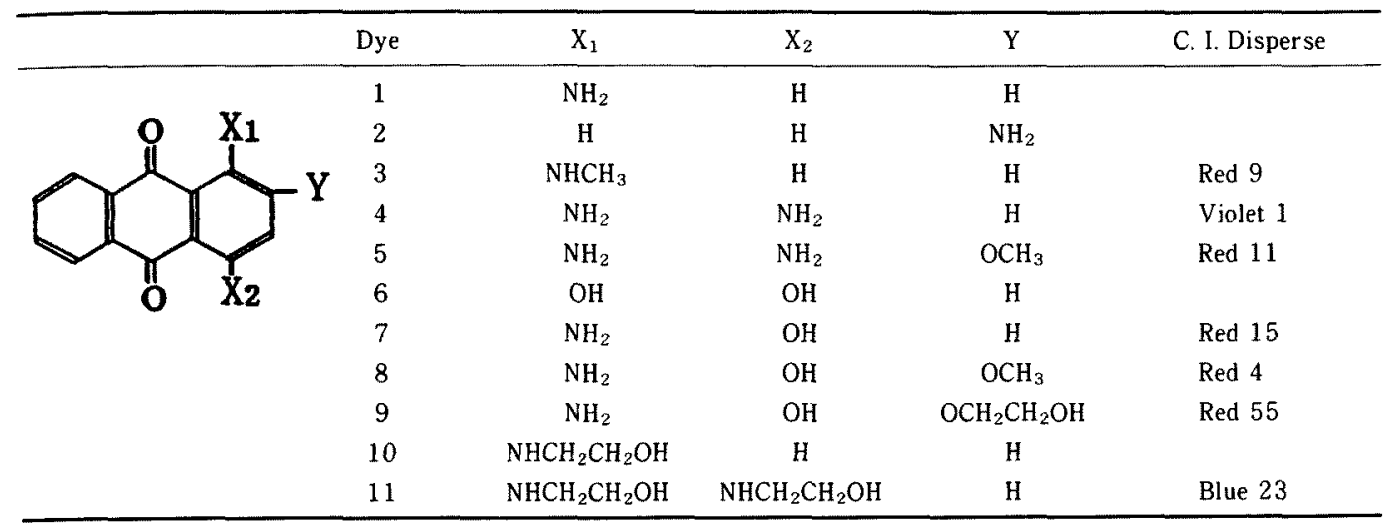

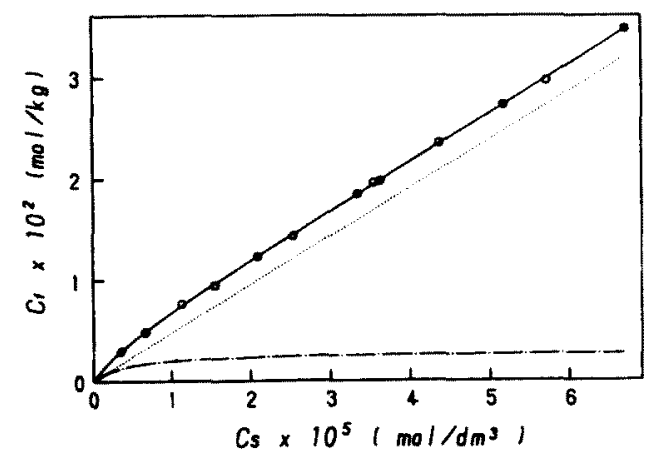

Fig. 1 Sorption isotherm of Dye 10 on Nylon 6 from water at $60^{\circ} \mathrm{C}: \bigcirc$. experimental point determined from dyeing in aqueous solution of dye, experimental point determined from dyeing in aqueous dispersion of dye. - calculated by Eq. 1 using the relevant values of $K_{P}$. $K_{L}$ and $S, \cdots \cdots$, partitioning isotherm, - . - . Lang. muir isotherm.

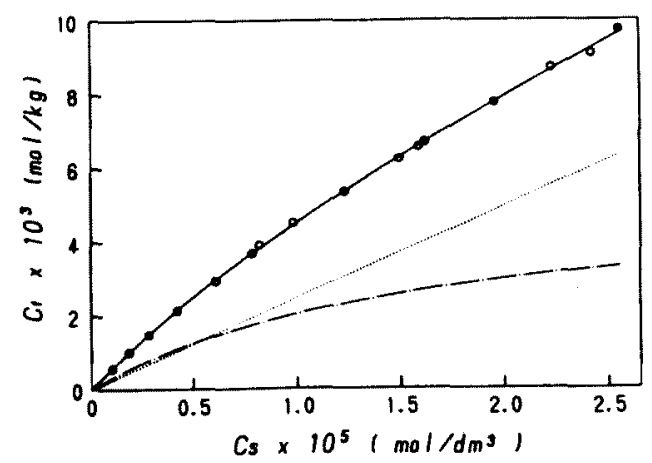

Fig. 2 Sorption isotherm of Dye 11 on Nylon 6 film at $60^{\circ} \mathrm{C}$, points and lines identified as in Fig. 1.

\subsection{Calculations}

Sorption isotherms were analyzed in terms of dual. mode sorption model as described before $[1,2]$. Three dimensional molecular model to minimize the energy of molecule in MM 2 forced field [3] was calculated using 'Chem 3D Plus' program (Cambridge Scientific Computing Inc.).

\section{Results and Discussion}

Figs. 1 and 2 show the sorption isotherms of Dyes 10 and 11 measured at $60^{\circ} \mathrm{C}$, respectively. For each isotherm, the plot of $C_{S}$ against $C_{F}\left[K_{L}+\left(1 / C_{S}\right)\right]$ fell on a straight line, indicating that these isotherms can be described by equation $1(1,2)$. The dual-sorption para. meters, $K_{\mathrm{p}}, \mathrm{K}_{\mathrm{L}}$ and $\mathrm{S}$ were estimated by nonlinear least squares fitting of the sorption data to Eq. 1. The experimental isotherms are separated into partitioning and Langmuir's as shown in Figs. 1 and 2 using the dual. sorption parameters; the separation explicitly reveals the contribution of each sorption mode to the dye sorption. The sorption isotherms observed for the other dyes at 60 and $80^{\circ} \mathrm{C}$ were similar to those shown in Figs. 1 and 2 . The values of $K_{p}, K_{1}$ and $S$ estimated are given in Table 2. The values of $C_{l} / C_{r}$ at saturated dye concentration in water calculated from the sorption parameters are listed in the last column of Table 2 . In addition to the value of $C_{i} / C_{r}$, the value of $S$ may also represent the extent of Langmuir sorption in the dual mode dye sorption [2]. For the azo disperse dyes with one $N$-substitued 2 . hydroxyethyl group (Disperse Orange 5 and Red 1) the values of $S$ markedly decreased with increasing temperature $\left(60 \rightarrow 80^{\circ} \mathrm{C}\right)[2]$, while those for Dyes $1.4,5.9$ and 11 
Table 2 Sorption Parameters

\begin{tabular}{|c|c|c|c|c|c|}
\hline $\begin{array}{l}\text { Dye } \\
\text { No. }\end{array}$ & $\begin{array}{c}\text { Temp. } \\
\left({ }^{\circ} \mathrm{C}\right)\end{array}$ & $\begin{array}{c}\mathrm{Kp} \\
\left(\mathrm{kg} / \mathrm{dm}^{3}\right) \\
\end{array}$ & $\begin{array}{l}\mathrm{K}_{\mathrm{L}} \times 10^{5} \\
\left(\mathrm{dm}^{3} / \mathrm{mol}\right)\end{array}$ & $\begin{array}{l}\mathrm{S} \times 10^{3} \\
(\mathrm{~mol} / \mathrm{kg})\end{array}$ & $C_{t} / C_{f}$ \\
\hline \multirow{2}{*}{1} & 60 & 709 & 6.48 & 2.46 & 0.139 \\
\hline & 80 & 305 & 1.24 & 2.41 & 0.120 \\
\hline 2 & 80 & 216 & 0.890 & 2.47 & 0.194 \\
\hline 3 & 80 & 598 & 5.63 & 1.49 & 0.0811 \\
\hline \multirow{2}{*}{4} & 60 & $703^{a 1}$ & 1.30 & 7.13 & 0.279 \\
\hline & 80 & 270 & 0.385 & 6.92 & 0.234 \\
\hline \multirow{2}{*}{5} & 60 & 1080 & 2.84 & 6.25 & 0.228 \\
\hline & 80 & 416 & 0.854 & 6.06 & 0.197 \\
\hline 6 & 80 & 390 & 21.1 & 0.357 & 0.0729 \\
\hline \multirow{2}{*}{7} & 60 & 1150 & 5.13 & 3.16 & 0.143 \\
\hline & 80 & 494 & 1.27 & 2.25 & 0.0747 \\
\hline 8 & 80 & 873 & 6.72 & 1.54 & 0.0979 \\
\hline \multirow{2}{*}{9} & 60 & 1340 & 6.26 & 3.11 & 0.181 \\
\hline & 80 & 462 & 1.18 & 3.39 & 0.152 \\
\hline 10 & 60 & 477 & 2.30 & 2.83 & 0.0767 \\
\hline \multirow{2}{*}{11} & 60 & 254 & 0.651 & 5.06 & 0.327 \\
\hline & 80 & 102 & 0.208 & 4.84 & 0.320 \\
\hline
\end{tabular}

a) Determined by omitting the sorption data in the range of very low dye concentration.

were almost constant irrespective of temperature. For Dye 7 , the decrease in $\mathrm{S}$ with increasing temperature was not so appreciable as in the cases of Disperse Orange 5 and Red 1 [2].

Figs. 1 and 2 clearly show that the value of $C_{1} / C_{f}$ for Dye 11 is much larger than that of Dye 10 over the almost whole range of $\mathrm{C}_{\varsigma}$. This result is concerned with the fact that Dye 11 has two hydroxy groups capable of inter-molecular $H$ bonding with nylon 6, whereas Dye 10 has only one. Furthermore, this is in accordance with the sorption behavior of azo disperse dyes [2].

It was reported that both hydroxy and amino groups introduced in 1 or 4-position of anthraquinone are largely involved in intramolecular H-bonding as shown in Fig. $3[4,5]$. On the other hand, the amino group substituted in 2-position is limited to form intermolecular H-bonding (3). It is known that the $H$-bonding between a carbonyl and a hydroxy group ( $\left.>\mathrm{C}=\mathrm{O}^{\circ} \mathrm{HO}^{-}\right)$is stronger than that between $>\mathrm{C}=\mathrm{O} \cdots \mathrm{H} \cdot \mathrm{NR} \cdot[4]$. Therefore the hydrogen atom of the hydroxy grope in 1 or 4-position has low capabiliy

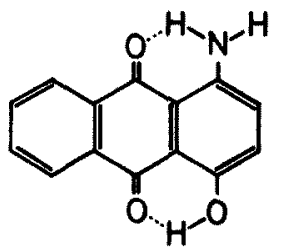

Fig. 3 Intramolecular H-bondings of 1-amino-4-hydroxy antraquinone.

to form intermolecular H-bonding. The hydrogen atom of l-methyl amino group may also be involved in intra. molecular $\mathrm{H}$-bonding, so that it has low capability to form intermolecular H-bonding. However, the primary amino group in 1 or 4-position, despite one of the hydrogen atoms involved in the chelation, still has another hydrogen atom capable of forming intermolecular $\mathrm{H} \cdot$ bonding.

It is noted that the values of $S$ and $C_{L} / C_{f}$ for Dye 4 are much larger than those for Dye 1. This is due to the fact that Dye 4 has two amino groups capable of intermolecu. lar H-bonding, while Dye 1 has one. The similar figure is 
<smiles></smiles>

Fig. 4 Intramolecular H-bonding between a hydrogen atom of 1 -amino group and an oxygen atom of a substituent at $2 \cdot$ position.

also found is the comparison of Dye 10 with Dye 11 as described above.

The values of $S$ and $C_{6} / C_{r}$ for Dye 3 are smaller than those for Dye 1 . This is also explained by considering the possibility of formation of $\mathrm{H}$-bonding between dye and substrate. Dye 1 has one hydrogen atom available to form intermolecular H-bonding, while Dye 3 has no such hydrogen atom owing to the chelation shown in Fig.3. For 1.4-disubstituted dye (Dye 4.6 and 7), the values of $S$ and $C_{L} / C_{r}$ are in the order of Dye $4>$ Dye $7>$ Dye 6 . This order is the same as the number of the hydrogen atoms the dyes acting as proton donor in intermolecular $H \cdot$ bonding.

For Dyes 5,8 and 9 , the additional formation of intramolecular $\mathrm{H}$-bonding as in Fig.4 is inferred from the dis. tance $r$. The values of $r$ were estimated from the three dimensional molecular model to minimize the energy of the molecules as follows ; Dye $5: 2.26 \dot{A}$, Dye $8: 2.26 \dot{A}$ and Dye $9: 2.25 \dot{A}$. These values of $r$ are less than $H$ bond length of $\mathrm{NH} \cdots \mathrm{O}, 2.89 \AA[6]$. The values of $\mathrm{S}$ and $\mathrm{C}_{\mathrm{L}} / \mathrm{C}_{\mathrm{f}}$ for Dye 5 are slightly smaller than those for Dye 4. This may be explained in terms of the additional formation of intramolecular H.bonding shown in Fig. 4.
This chelation reduces the capability of the amino group to form intermolecular $\mathrm{H}$-bonding, resulting in the reduction of the contribution of Langmuir sorption. Similar situation is observed for the difference in the $S$ value between Dye 7 and 8 . Although Dye 9 also forms such additional chelation as shown in Fig. 4, however, the values of $C_{1} / C_{f}$ for this dye are larger than that Dye 7 . This may be ascribed to the fact that the Hydrogen atom in 2-hydroxyethoxy group is capable to form inter. molecular $\mathrm{H}$-bonding.

This findings described above indicate that in the sorption of anthraquinone disperse dye by nylon 6 from water, the contribution of Langmuir sorption to the total dye uptake increases with an increase in the number of the substituents capable of forming intermolecular $\mathrm{H}$. bonding or the capability of the hydrogen atoms of substituents to form intermolecular H-bonding. This suggests that $\mathrm{H}$-bonding between the dyes and Nylon 6 plays an important role in Langmuir sorption, where the dyes act as proton donors.

\section{References}

1. T. Shibusawa and Y. Chigira, J. Polym. Sci., Part B. Polym. Phys., 30, 563 (1992).

2. T. Shibusawa and N. Arai, H. Ueno and T. Nakamura, Sen'i Gakkaishi, 48, 641 (1992).

3. U. Burkert and N. L. Allinger. 'Molecular Mechanics'. Amer. Chem. Soc., Washington DC, (1982).

4. R. H. Peters and H. H. Sumner, J. Chem. Soc. 2101 (1953).

5. M. St. C. Flett, J. Chem. Soc., 1441 (1948).

6. L. N. Kuleshova and P.M. Zorkii. Acla Crystallogr... B37, 1363 (1981). 